\title{
SHORT PROOF OF A MAP-COLOUR THEOREM
}

\author{
G. A. DIRAC
}

Heawood (3) showed that for $h>1$ the countries of any map on a surface of connectivity $h=3-\chi$ can be coloured using at most

$$
n_{h}=\left[\frac{1}{2}(7+\sqrt{ }(24 h-23))\right]
$$

colours. In a previous paper (1) I proved the following

THEOREM. For $h=3$ and for $h \geqslant 5$ a map on a surface of connectivity $h$ with chromatic number $n_{h}$ always contains $n_{h}$ mutually adjacent countries.

A new short proof of this will now be given. It is based on the following result (2):

If a critical $k$-chromatic graph contains $N>k$ nodes and $E$ edges then

$$
2 E \geqslant(k-1) N+k-3 \text {. }
$$

(A graph is called critical if deleting any arbitrary node or edge reduces the chromatic number. A $k$-chromatic graph always contains a critical $k$-chromatic subgraph, and a critical graph is finite and connected (1, p. 481).)

To prove the above map-colour theorem it is sufficient to show that for $h=3$ and $h \geqslant 5$, if a critical $k$-chromatic graph with more than $k$ nodes is drawn without intersection of edges on a surface of connectivity $h$, then $k \leqslant n_{h}-1$. Let such a graph have $N$ nodes and $E$ edges. By (1) and Euler's Theorem (1, 2.1), we have

$$
(k-1) N+k-3 \leqslant 2 E \leqslant 6 N+6(h-3) .
$$

Further $(1,1.3), N \geqslant k+2$.

For the case when $h=3$, we observe that $n_{3}=7$ and, from (2), $k \leqslant 6$. For $h \geqslant 5$ it may be assumed that $k>6$, so it follows from (2) that

$$
(k-7)(k+2) \leqslant(k-7) N \leqslant 6 h-15-k,
$$

whence

$$
k \leqslant 2+[\sqrt{ }(6 h+3)] .
$$

Thus $k \leqslant n_{h}-1$ when

$$
[\sqrt{ }(6 h+3)] \leqslant\left[\frac{1}{2}(1+\sqrt{ }(24 h-23))\right] .
$$

This is clearly true if

$$
\sqrt{ }(6 h+3) \leqslant \frac{1}{2}(1+\sqrt{ }(24 h-23)),
$$

that is, if $h \geqslant 13$. It can be verified also for $h=5,6, \ldots, 12$. This completes the proof of the Theorem.

Received December 31, 1956. 


\title{
ReFERENCES
}

1. G. A. Dirac, Map-colour theorems, Can. J. Math., \& (1952), 480-490.

2. - A theorem of R. L. Brooks and a conjecture of H. Hadwiger, Proc. London Math. Soc. (3), 7 (1957).

3. P. J. Heawood, Map-colour theorem, Quart. J. Math., 24 (1890), 332-338.

Vienna XIX (Hardtgasse 19), Austria

\section{GORRECTION TO "A MINIMUM-MAXIMUM PROBLEM FOR DIFFERENTIAL EXPRESSIONS"}

\author{
D. S. CARTER
}

The author takes this opportunity to correct some misprints and to add a note to his paper "A minimum-maximum problem for differential expressions," in this Journal, 9 (1957), 132-140.

Page 134, Equation (2.5): for this equation read

$$
\left\|\mathfrak{R} x_{0}\right\|=\inf \{\|\mathfrak{R}\| \| x \in X\} \text {. }
$$

Page 137, line -5 : for " $e_{0}^{i}$ " read " $e$ ".

Page 138, line -7 : for " $\left|\xi_{0}^{i}\right|$ " read " $\left|\zeta_{0}\right| "$.

Added note: Since the preparation of this manuscript it has come to the author's attention that the present problem bears a close relationship to the "Bang-Bang" control problem (3). Choosing $c=0, \eta_{b}=0$, and allowing the endpoint $b$ to vary, it is easy to show that the value of $\left\|g_{0}\right\|$ at the solution is a continuous monotone function of $b$. The value of $b$ for which $\left\|g_{0}\right\|=1$ provides the solution to a "Bang-Bang" problem of a rather general type.

\section{REFERENCE}

3. R. Bellman, I. Glicksburg, and O. Gross, On the "Bang-Bang" Control Problem, Quart. Appl. Math. 14 (1956), 11-18.

Los Alamos Scientific Laborotory 